\title{
Correction to: Bacterial Community of the Digestive Tract of the European Medicinal Leech (Hirudo verbana) from the Danube River
}

\author{
Saraswoti Neupane $^{1}$ - David Modry ${ }^{2,3} \cdot$ Barbora Pafčo $^{2} \cdot$ Ludek Zurek $^{1,2,3}$ (D)
}

Published online: 26 March 2019

(C) Springer Science+Business Media, LLC, part of Springer Nature 2019

\section{Correction to: Microbial Ecology \\ https://doi.org/10.1007/s00248-019-01349-Z}

The original published version of this article had mistakes in figure legends. Correct figure legends are presented below.

Fig. 1 Bacterial $\alpha$-diversity (Shannon diversity index) in gut of wild medicinal leeches. LSMEANS $+/-95 \%$ confidence intervals of Shannon diversity index are shown for $n=3$ gut positions, where, $\mathrm{CA}=$ anterior crop, $\mathrm{CP}=$ posterior crop and $\mathrm{I}=$ intestine

Fig. 3 Relative abundance of dominant bacterial phyla and genera in individual leeches. Gut position: $\mathrm{CA}=$ anterior crop, $\mathrm{CP}=$ posterior crop and $\mathrm{I}=$ intestine. Samples from three different leeches (L1, L2 and L3) are colored in blue, magenta and orange respectively

Fig. 4 Principal Co-ordinates Analysis (PCoA) of bacterial diversity based on Bray-Curtis distance. Samples from three different leeches (L1, L2 and L3 are colored in blue, magenta and orange respectively). The Gut Position is the position of sample in gastrointestinal tract: anterior crop (CA), posterior crop (CP) and intestine (I)

The online version of the original article can be found at https://doi.org/ 10.1007/s00248-019-01349-Z

Ludek Zurek

lzurek01@gmail.com

1 Department of Entomology, Kansas State University, Manhattan, KS, USA

2 Department of Pathology and Parasitology, University of Veterinary and Pharmaceutical Sciences, Brno, Czech Republic

3 Central European Institute of Technology, Brno, Czech Republic 